\title{
Author Correction: Investigation of Dietary Factors and Esophageal Cancer Knowledge: Comparison of Rural Residents in High- and Low- incidence Areas
}

\begin{abstract}
Dong Tian ${ }^{1}$, Shuai-Jia Mo², Lian-Kui Han ${ }^{3}$, Liang Cheng ${ }^{1}$, Heng Huang ${ }^{2}$, Shuai Hao ${ }^{2}$, Ye-Lan Guan'2, Kai-Yuan Jiang ${ }^{2}$, Jing-Ya Deng ${ }^{2}$, Hu-Hao Feng ${ }^{2}$, Hong-Ying Wen ${ }^{1}$ \& Mao-Yong Fu ${ }^{1}$

Correction to: Scientific Reports https://doi.org/10.1038/s41598-018-23251-3, published online 20 March 2018
\end{abstract}

This Article contains errors in the Introduction section.

"This survey was administered in Yanting County of Guizhou Province, which is a high-incidence area for EC, and in Qingzhen City, which is a low-incidence area. Yanting, a rural and one the most indigent counties in Sichuan Province, is located northeast of Chengdu City, at $105^{\circ}$ latitude N and $31^{\circ}$ longitude E."

should read:

“This survey was administered in Yanting County of Sichuan Province, which is a high-incidence area for EC, and in Qingzhen City of Guizhou Province, which is a low-incidence area. Yanting, a rural and one the most indigent counties in Sichuan Province, is located northeast of Chengdu City, at $31^{\circ}$ latitude N and $105^{\circ}$ longitude E."

(C) The Author(s) 2018

\footnotetext{
${ }^{1}$ Department of Cardiothoracic Surgery, Affiliated Hospital of North Sichuan Medical College, Nanchong, 637000, China. ${ }^{2}$ College of Basic Medicine, North Sichuan Medical College, Nanchong, 637000, China. ${ }^{3}$ Department of Thoracic Surgery, Guizhou Provincial People's Hospital, Guiyang, 550002, China. Dong Tian, Shuai-Jia Mo and Lian-Kui Han contributed equally to this work. Correspondence and requests for materials should be addressed to H.-Y.W. (email: why-1166@163.com) or M.-Y.F. (email: fumaoyongmd@126.com)
} 